\title{
Thermodynamic Study of Micellization of SDBS in Aqueous and in Binary Solvent Systems of Ethylene Glycol
}

\author{
Madhu Khandelwal ${ }^{1 *}$,Dr. Ampily. J.S ${ }^{2}$, Dr. Bijendra Rai ${ }^{1}$ and Dr. Geetha Sarasan ${ }^{1}$ \\ ${ }^{1}$ Department of Chemistry, Holkar Science College, Indore (India). \\ ${ }^{2}$ Department of Chemistry, R. Sankar Memorial SNDP Yogam Arts and Science College, Koyilandy, \\ Calicut, Kerala (India),
}

\begin{abstract}
Knowledge of Micellar behavior of surfactants in aqueous and in co-solvent system is essential for wide range of applications. Association behavior of an anionic surfactant, Sodium dodecylbenzenesulphonate (SDBS) has been studied conductometrically at different temperatures in aqueous and in various compositions of ethylene glycol (EG) and water. The critical micelle concentration (CMC) and degree of counter-ion dissociation values $(\alpha)$ of SDBS increase as the mole fraction of ethylene glycol (EG) increase at constant temperature and also increase with increasing temperature at constant mixture composition. Phase separation model, which gives quantitative theoretical interpretation of thermodynamic parameters of micellization to evaluate the free energy, enthalpy and entropy of micellization over the temperature range of 298-318K. The thermodynamic analysis shows that, although the micellization is less favorable in mixed solvent compared to pure water but the process is spontaneous and exothermic. It was found that the free energy of micellization values become less negative whereas the enthalpy values becomes more negative in binary solvent systems than aqueous solution. The $\Delta S^{0}$ values for aqueous solution of SDBS are higher than that of binary solvent systems that micellization is favoured by entropy gain in aqueous solution.
\end{abstract}

Key words: Micellization, SDBS, Ethylene glycol, Thermodynamics.

\section{INTRODUCTION}

In recent years there has been a great interest on the study of self assembly properties of surfactant in solvent medium containing a binary mixture of water and polar/non polar solvents $[1,2,3,4]$. It is well known that surfactant molecules are associated into micelles above the critical micelle concentration (CMC) and self association of surfactant is dependent on many factors but largely dependent on nature of the solvent as well as temperature of the solution. Inclusion of different types of solvents influences the micellar properties by affecting the solvent structure or morphology. The addition of co-solvent in aqueous medium is of considerable interest since the applications of surfactants in industrial process like cleaning operation, lubrication, oil-wetting cleaners, etc., which require water free or poor water medium [5]. Aggregation behavior of surfactant in solvents other than water (non aqueous) is considerably dependent on solvent polarity and its ability to form hydrogen bond in solution. Micellization can be possible in solvents such as ethylene glycol, hydrazine, formamide, glycerol, which have high degree of hydrogen bonding $[6,7,8,9]$. On the other hand micelle formation is also possible in solvents like acetone, acetonitrile and dimethylsulfoxide where the hydrogen bonding ability is less [10,11]. Beside hydrogen bonding ability, hydrophobicity of the solvent also plays a significance role in micellization process. Micellization process of ionic and non ionic surfactants in presence of co-solvent has been studied by many investigators. A.D. Fenta investigated the surface and thermodynamic studies of surfactant (SDS, HTAB and Tween 80) in binary mixtures of 1, 2 -ethanediol (EG) and 1, 2, 3-propanetriol (PG) with water by applying conductance, surface tension and dye solubilization (UV-Vis absorption spectroscopy) techniques [12]. In another thermodynamic study K. Prajapati and S. Patel have been studied the micellization of SDS, $\mathrm{C}_{16} \mathrm{TABr}$, and Tween 80 in EG/water and formamide-water mixed solvent using surface tension, viscosity and conductance measurements [13]. K. Gracie et. al. has been reported the micellar properties of Sodium dodecylsulphate (SDS) in aqueous binary mixtures of ethylene glycol (EG) using various techniques such as conductivity, density, EMF, surface tension, viscosity, ultrasonic velocity and spectroscopy (fluorescence). They were also calculated the thermodynamics of micellization from the temperature dependence of CMC values [7]. SE Olaseni et. al. has been investigated the micellization behaviour of Cetyltrimethylammonium bromide (CTABr) in ethylene glycol (EG), dimethylsulfoxide (DMSO) and dimethylformamide (DMF)-water mixed solvents, using electrical conductivity measurement at different temperatures[11].

Glycols are well-known for their use as coolants and antifreezes and find application in pharmaceutical, cosmetics, and food industries [14]. In most these applications, surfactant is also present and therefore it is important to study the effect of glycol on micellization of surfactant.

\section{MATERIALS AND METHOD}

Anionic surfactant, SDBS purchased from Sigma Aldrich and used as received. Ethylene glycol is a polar, colorless, viscous liquid, obtained from Merck Pvt. Ltd. and used without further purification. Conductivity water was used for preparing all solutions. 


\section{Conductance measurements:}

Concentrated stock solutions ( 10 to15 times the CMC) of SDBS were prepared individually in pure aqueous and in different compositions of binary solvent of EG with water. The conductivity measurements were performed with digital Systronics conductivity meter (306). Initially $25 \mathrm{ml}$. of solvent (water/ binary mixture of solvent) was placed in a beaker and concentrated surfactant solution (prepared in the desired solvent medium) of known concentration was progressively added in solvent. The temperature of the solution was maintained by temperature controlled circulator water bath, holding the solution under study to maintain the desired temperature. The specific conductance of the solution was observed after each addition followed mixing on magnetic stirrer and attaining temperature equilibrium of system. The procedure was repeated for different temperatures and CMC of SDBS was obtained from the break point in the conductance versus concentration plot.

\section{Effect of Ethylene glycol on CMC and degree of ionization:}

\section{RESULTS AND DISCUSSION}

The specific conductance of SDBS was observed from pre micellar concentration to post micellar concentration in aqueous and in binary solvent systems of EG $(5 \%, 15 \%$ and $25 \%)$ with water at $298 \mathrm{~K}$. The specific conductance plotted against molar concentration of surfactant and the concentration corresponding to the intersection of these two lines gives the CMC (i.e. point of aggregation) of SDBS (Figure 1). The CMC value of SDBS in pure aqueous medium obtained are in good agreement with literature values [15]. The ratio of the slopes of post micellar region to pre micellar region gives the degree of ionization $(\alpha)$ of the surfactant. It has been observed that when the solvent system changes from aqueous to binary solvent systems at specific temperature, the CMC and degree of ionization values of SDBS increase (Table 1). The increase of CMC with ethylene glycol concentration can be explained on the basis of change in the bulk phase properties (e.g. dielectric constant, dipole moment, viscosity, etc.) of the solvent system [16]. On addition of EG fractions in aqueous solution causes decrease of the dielectric constant of binary solvent system because EG dielectric constant [ $E=37.7]$ is lower than water[ $E=78.54]$, so lowering of dielectric constant would result in an increase in the repulsion between the ionic head groups leading to delayed micellization. Ethylene glycol has a high degree of hydrogen bonding (presence of two-OH groups) and act as a water structure breaker (ability to form intermolecular H-bonds with water) [17]. It would facilate the interaction between the hydrocarbon part of organic solvent and hydrophobic tail of the surfactant molecule. Hence difficult for the hydrocarbon chain of surfactant to aggregate due to solvation of them and so the lesser number of monomer to form micelles and hence increase the CMC of surfactant [18]. An increase in degree of ionization with respect to solvent percentage is expected due to decrease charge density on the micellar surface caused by a reduction of the micellar aggregation number [19]. Another fact is viscosity of the medium also increases on increasing the \% of EG in water and it becomes difficult for surfactant tail to migrate easily from bulk phase to the micellar phase result in the increase of CMC of surfactant.

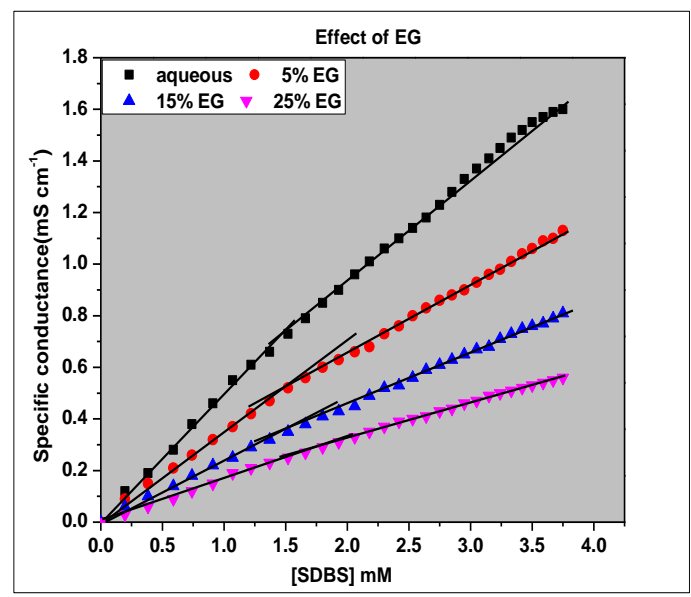

Figure 1: Plots of specific conductance Vs molar concentration of SDBS in various compositions of EG and water at $298 \mathrm{~K}$

\section{Effect of temperature on CMC and degree of ionization:}

The plots of specific conductance and molar concentration of SDBS for aqueous and binary solvent systems at different temperatures are presented in Figure 2. The CMC values of SDBS in water and in binary solvent systems $(5 \%, 15 \%$ and $25 \%)$ of EG and water increase with increase of temperature (Table 1, Figure 3). Increased thermal motion on increasing the temperature is responsible for the disruption of the water structure surrounding the alkyl tail part of ionic surfactants (which opposes the micellization) and inhibits the formation of ordered structure of micelles [11,20]. Another fact is the dehydration of surfactant ionic head groups at elevated temperatures, resulting in a stronger repulsion of their ionic heads which disfavor the formation of micelles [21]. The degree of ionization value also increase with temperature (Table 1 Figure 4) and it could be attributed to the fact that thermal forces increases the separation between the ionic head groups and counter ions[11]. 


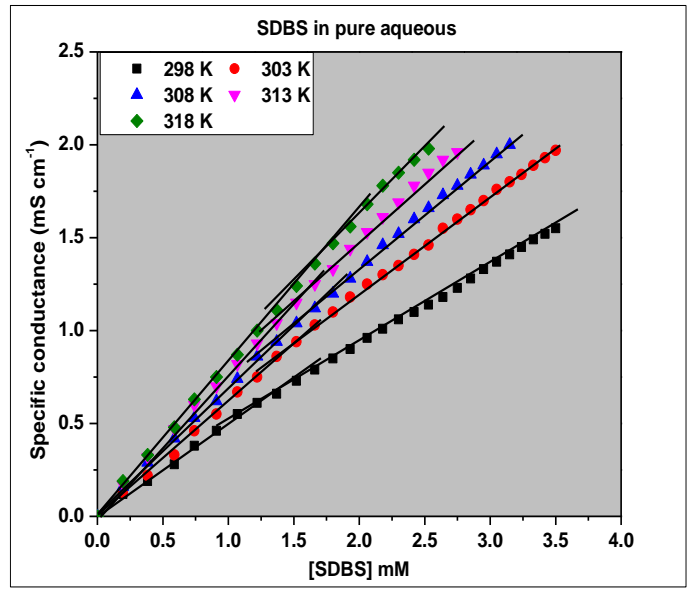

Figure (a)

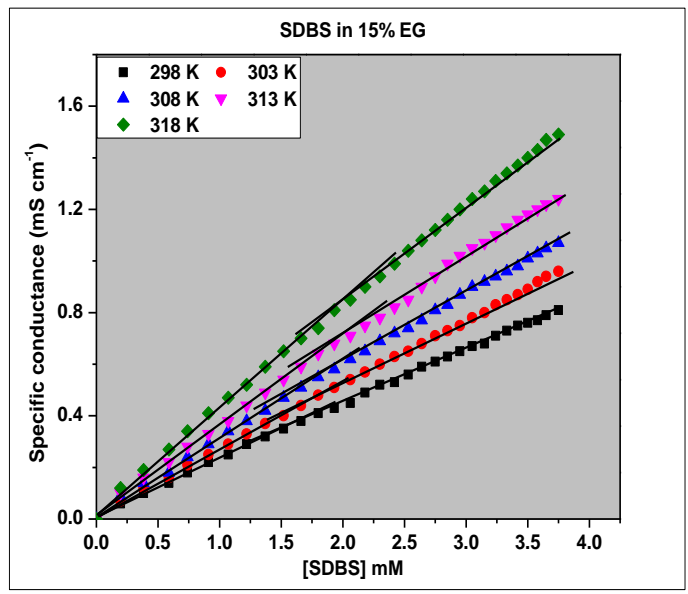

Figure (c)

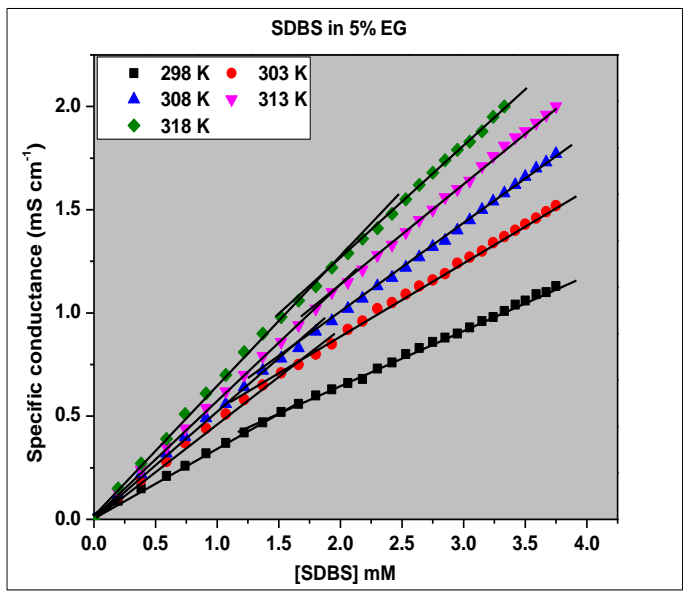

Figure (b)

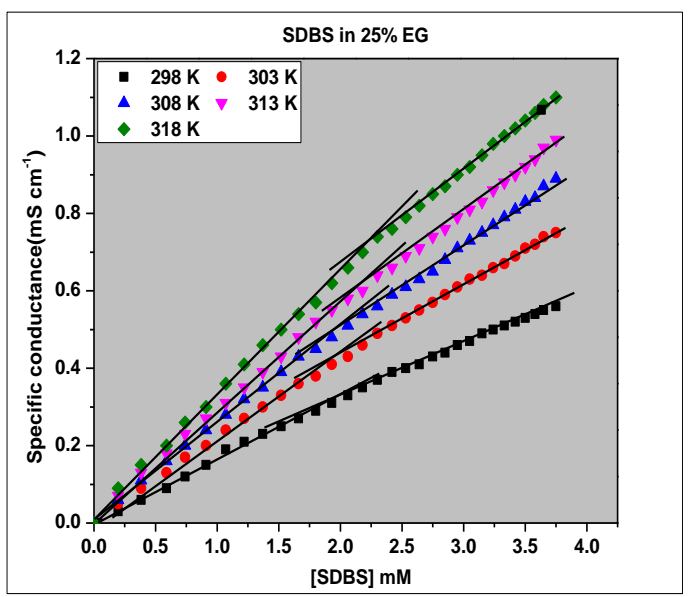

Figure (d)

Figure 2: Plots of specific conductance Vs molar concentration of SDBS in aqueous and in binary solvent systems of EG and water at different temperatures

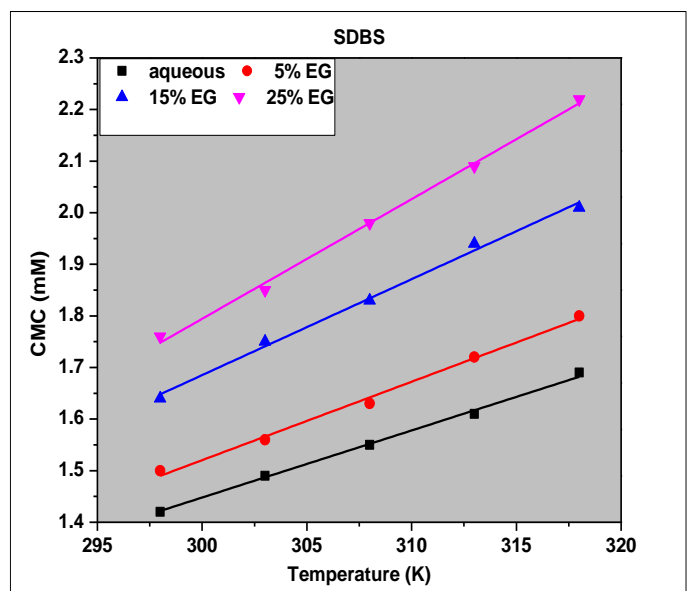

Figure 3

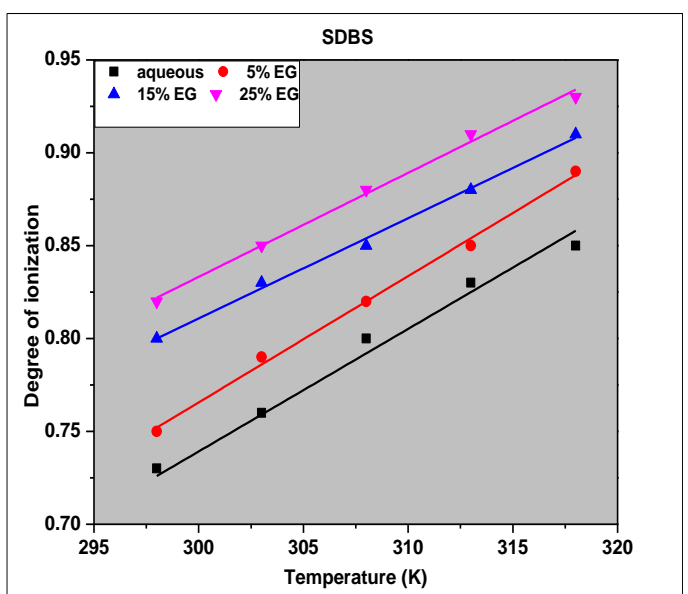

Figure 4

Figure 3: Effect of temperatures on CMC of SDBS in aqueous and in binary solvent systems

Figure 4: Effect of temperatures on degree of ionization of SDBS in aqueous and in binary solvent systems 
Table 1: CMC and degree of ionization $(\alpha)$ of SDBS in aqueous and in binary solvent systems of EG and water at different temperatures

\begin{tabular}{|c|c|c|c|c|c|c|c|c|c|c|}
\hline \multirow[t]{3}{*}{ Solvent } & \multicolumn{10}{|c|}{ Temperature (K) } \\
\hline & \multicolumn{2}{|c|}{298} & \multicolumn{2}{|c|}{303} & \multicolumn{2}{|c|}{308} & \multicolumn{2}{|c|}{313} & \multicolumn{2}{|c|}{318} \\
\hline & CMC (mM) & $\alpha$ & $\mathrm{CMC}(\mathrm{mM})$ & $\alpha$ & $\mathrm{CMC}(\mathrm{mM})$ & $\alpha$ & CMC (mM) & $\alpha$ & $\begin{array}{l}\text { CMC } \\
(\mathbf{m M})\end{array}$ & $\alpha$ \\
\hline Water & 1.42 & 0.73 & 1.49 & 0.76 & 1.55 & 0.80 & 1.61 & 0.83 & 1.69 & 0.85 \\
\hline $5 \%$ EG & 1.50 & 0.75 & 1.56 & 0.79 & 1.63 & 0.82 & 1.72 & 0.85 & 1.80 & 0.89 \\
\hline $15 \%$ EG & 1.64 & 0.80 & 1.75 & 0.83 & 1.83 & 0.85 & 1.94 & 0.88 & 2.01 & 0.91 \\
\hline $25 \%$ EG & 1.76 & 0.82 & 1.85 & 0.85 & 1.98 & 0.88 & 2.09 & 0.91 & 2.22 & 0.93 \\
\hline
\end{tabular}

\section{Thermodynamics of micellization:}

Temperature dependence CMC values are used to evaluate thermodynamic parameters [Free energy of micellization $\left(\Delta G_{m}^{\circ}\right)$, Free energy of surfactant tail transfer $\left(\Delta G_{\text {trans }}^{o}\right)$, enthalpy of micellization $\left(\Delta H_{m}^{o}\right)$ and entropy of micellization $\left.\left(\Delta S_{m}^{o}\right)\right]$ of ionic surfactant and analyzed in terms of the phase separation model for micelle formation [22]. According to this model for ionic surfactant the standard Gibb's free energy of micellization are calculated from the following equation

$$
\Delta G_{m}^{\circ}=(2-\alpha) R T \ln X_{c m c}
$$

Where, $\mathrm{R}, \mathrm{T}$ and $X_{c m c}$ are ideal gas constant, absolute temperature and CMC expressed in mole fraction unit respectively. The corresponding enthalpy of micellization obtained from the following equation

$$
\text { a) } \mathrm{RT}^{2} \frac{d \ln X c m c}{d T}
$$

The values of $\Delta H_{m}^{o}$ calculated by substituting the value of slope of the curve between $\ln X_{c m c}$ versus temperature (Figure 5). Further the entropy of micelle formation calculated using the equation

$$
\Delta S_{m}^{o}=\frac{\Delta H_{m}^{o}-\Delta G_{m}^{o}}{T}
$$

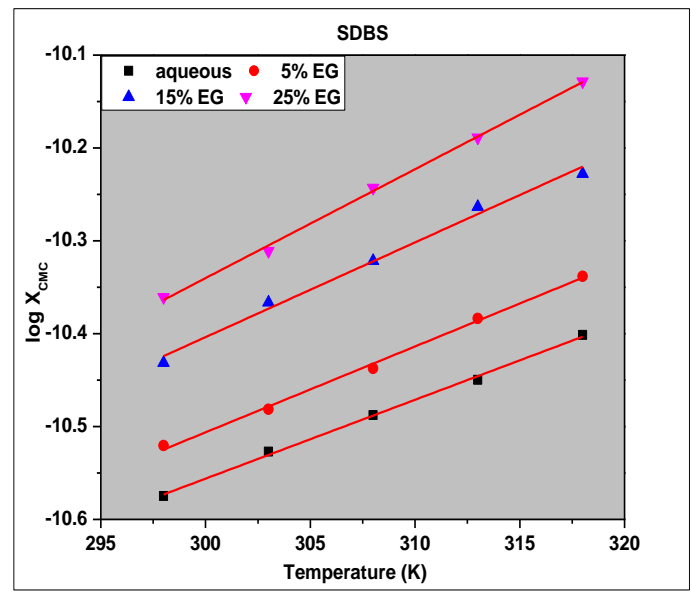

\begin{tabular}{|c|c|c|c|c|c|}
\hline \multirow[t]{2}{*}{ Co-solvent } & \multicolumn{5}{|c|}{ Temperature(K) } \\
\hline & 298 & 303 & 308 & 313 & 318 \\
\hline & \multicolumn{5}{|c|}{ Change in Gibbs free energy $\quad \Delta G_{m}^{\circ}\left(\mathrm{kJ} \mathrm{mol}^{-1}\right)$} \\
\hline water & -33.275 & -32.884 & -32.227 & -31.816 & -31.624 \\
\hline 5\%EG & -32.581 & -31.948 & -31.538 & -31.074 & -30.339 \\
\hline 15\%EG & -31.013 & -30.553 & $-\mathbf{- 3 0 . 3 9 5}$ & -29.913 & -29.474 \\
\hline \multirow[t]{2}{*}{ 25\%EG } & -30.289 & -29.870 & -29.376 & -28.900 & -28.652 \\
\hline & \multicolumn{5}{|c|}{ Change in enthalpy of micellization $\Delta H_{m}^{o}=\left(\mathrm{kJ} \mathrm{mol}^{-1}\right)$} \\
\hline water & -7.970 & -8.045 & -8.044 & -8.100 & -8.218 \\
\hline 5\%EG & -8.536 & -8.543 & -8.608 & -8.664 & -8.632 \\
\hline 15\%EG & -9.037 & -9.109 & -9.251 & -9.305 & -9.347 \\
\hline \multirow[t]{2}{*}{ 25\%EG } & -10.193 & -10.270 & -10.335 & -10.387 & -10.525 \\
\hline & \multicolumn{5}{|c|}{ Change in entropy of micellization $\Delta S_{m}^{o}\left(\mathrm{~J} \mathrm{~K}^{-1} \mathrm{~mol}^{-1}\right)$} \\
\hline water & 84.916 & 81.976 & 78.516 & 75.769 & 73.603 \\
\hline $5 \%$ EG & 80.687 & 77.244 & 74.448 & 71.599 & 68.261 \\
\hline $15 \%$ EG & 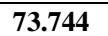 & 70.772 & 68.649 & $\mathbf{6 5 . 8 3 7}$ & 63.292 \\
\hline $25 \%$ EG & 67.436 & 64.686 & 61.821 & $\mathbf{5 9 . 1 4 7}$ & 57.003 \\
\hline
\end{tabular}

Figure 5: Plots of $\log \mathrm{X}_{\mathrm{CMC}}$ of SDBS with temperature for various solvent systems

Table-2: Thermodynamic parameters of SDBS in aqueous and in binary solvent systems at different temperatures 
The values of free energy of micellization $\left(\Delta G_{m}^{\circ}\right)$ are found negative $\left(\Delta G_{m}^{\circ}<0\right)$ for SDBS in aqueous as well as in binary solvent systems at different temperatures. It could be due to increase of CMC with temperature in both systems (aqueous and binary solvent systems) studied. The fractions of EG increases at specific temperature, the micellization process become less spontaneous (Table 2). The addition of glycol decrease the dielectric constant of medium and that result in the decrease of aggregation number hence surfactant monomers in bulk phase of solution increases. So that the degree of ionization $(\alpha)$ values and CMC values increase which further decrease the negative value of $\Delta G_{m}^{\circ}$ (Eq. 1). Another fact for the free energy of micellization becomes less negative in binary solvent systems of EG is that there may be different types of free energy associated with micellizaton process. It is mainly controlled by surfactant tail transfer $\left(\Delta G_{\text {trans }}^{o}\right)$ that accounted for the effect of co-solvent on the micellization process [23], which is defined by the expression $\Delta G_{\text {trans }}^{o}=\Delta G_{m(E G+H 2 O)}^{o}-\Delta G_{m(H 2 O)}^{o}$ [4]

Table 3: Effect of co-solvent on free energy of surfactant tail transfer $\left(\Delta G_{\text {trans }}^{o}\right)$

\begin{tabular}{|c|c|c|c|}
\hline Temperature $(\mathbf{K})$ & \multicolumn{3}{|c|}{ Free energy of surfactant tail transfer $\left(\Delta G_{\text {trans }}^{\boldsymbol{o}}\right)\left(\mathrm{kJ} \mathrm{mol}^{-1}\right)$} \\
\hline & $5 \%$ EG & $15 \%$ EG & $25 \%$ EG \\
\hline 298 & 0.69 & 2.26 & 2.99 \\
\hline 303 & 0.94 & 2.33 & 3.01 \\
\hline 308 & 0.69 & 1.83 & 2.85 \\
\hline 313 & 0.74 & 1.90 & 2.91 \\
\hline 318 & 1.28 & 2.15 & 2.98 \\
\hline
\end{tabular}

It is found that Free energy of surfactant tail transfer $\left(\Delta G_{\text {trans }}^{o}\right)$ are positive and increase with increasing solvent fractions (EG) in the micellar system at specific temperature (Table 3). It indicates that micellization is less spontaneous in binary solvent systems, thus apparent that transfer of surfactant hydrophobic tail from bulk phase to micelle phase is less spontaneous.

The values of enthalpy of micellization $\left(\Delta H_{m}^{o}\right)$ are found to be negative and becomes more negative with increasing temperature as well as with increase in the volume fraction of EG in aqueous medium (Table 2). It is clear that the enthalpy change favors the process of micellization and proceeds via an exothermic process. The negative $\Delta H_{m}^{o}$ is associated with the hydrophobic interaction in the interior of the micelle with the release of more arranged water molecules (or melting of the "iceberg") surrounded to hydrophobic tail of the monomer. It becomes more exothermic as the temperature increases because at higher temperature the hydrogen bond between the water molecules is diminished. Consequently, less energy is required to break up the iceberg water structure around hydrophobic alkyl chains [24]. The enthalpy of micellization is found more negative as the EG content increases due to formation of more intermolecular hydrogen bonds between EG and water. The results of entropy of micellization for SDBS are given in Table 2 and found that $\Delta S_{m}^{o}$ values are positive for each solvent system, indicates that the micellization is favored by entropy gain. The positive value of $\Delta S_{m}^{o}$ indicates that the micellization process is governed with the destruction of the iceberg around the hydrophobic alkyl chain. The decrease of $\Delta S_{m}^{o}$ values with increasing temperature in aqueous as well as in binary solvent systems, suggest that disordering of water molecules becomes less pronounced with increasing temperature because as temperature increases, the "structured water layer" around the hydrophobic tail of a monomer becomes thinner (degree of structure in the water layer decrease). Therefore the extent of randomness decreases with increasing temperature [24]. Although the magnitude of $\Delta S_{m}^{o}$ is lower in the presence of co-solvent compare with aqueous surfactant solution, but positive value of entropy indicates that the solvent (EG) still control the 3-dimensional water structure and increase orderliness of water by intermolecular hydrogen bonding with ethylene glycol [11]. It can be attributed from the results of study that the micellization process is favoured by enthalpy gain at higher temperature and entropy driven at lower temperature.

\section{CONCLUSION}

Investigation carried out in surfactant-binary solvent systems has considerable attention due to their wide range of applications in water free or poor water medium. Both the addition of co-solvent (Ethylene glycol) and rise in temperature results in an increase in the critical micelle concentration (CMC) and degree of ionization for studied surfactant. Due to change in bulk phase properties of binary solvent systems are responsible for unfavourable micellization in binary solvent systems. Thermal agitation causes disruption of water molecules surrounding the alkyl tail part of surfactant and hence the CMC increases for both systems. According to thermodynamics of micellization decrease in free energy of system suggesting micellization is spontaneous and favourable but increase in temperature and addition of EG in water decreases the spontaneity of micellization of SDBS surfactant. Micellization is favoured by enthalpy change at higher temperature and entropy gain at lower temperature. Knowledge of CMC and thermodynamics of micellization are essential for understanding spontaneity and stability. It would be beneficial for various types of applications like cleaning, lubrications, pharmaceutical, cosmetic, petroleum and mineral processing etc. which require lesser amount of water. 


\section{REFERENCES}

[1] A.Rodriguez, M.M.Gracian and M.L.Moya, "Effects of addition of polar organic solvents on micellization." Langmuir, vol. 24, 12785-12792, 2008.

[2] S.Das, S.Mondal and S.Ghosh, "Physicochemical Studies on the Micellization of Cationic, Anionic and Nonionic Surfactants in Water-Polar Organic Solvent Mixtures," J. Chem. Eng. Data, vol. 58, no. 9, pp. 2586-2595, 2013.

[3] K.K Ghosh and V. Baghel, "Micellar properties of benzyldimethylammonium bromide in aquo- organic solvent media," Indian J. Chem., vol. 47A, pp. 1230-1233, 2008.

[4] Kabir-ud-Din, U. S. Siddiqui, S. Kumar and A. A. Dar, "Micellization of monomeric and dimeric (gemini) surfactants in polar non aqueous-watermixed solvents," Colloid Polym. Sci., vol. 284, no. 7, pp. 807-812, 2006.

[5] C. Carnero Ruiz, "Thermodynamics of micellization of tetradecyltrimethylammonium bromide in ethylene glycol-water binary mixtures," Colloid Polym. Sci., vol. 277, 701-707 1999.

[6] S.S. Kumar and N. Rathod, "Micellar Properties of Alkyltrimethyl Ammmonium Bromide in Aquo-organic Solvent Media," Res. J. Chem. Sci., vol. 1, no. 4, pp. 22-29, 2011.

[7] K. Gracie, D. Turner and R. Palepu, "Thermodynamic properties of micellization of Sodium dodecyl sulfate in binary mixtures of ethylene glycol with water," Can. J. Chem., vol. 74, no. 9, pp. 1616-1625, 1996.

[8] R. Palepu, H.Gharibi, D.M. Bloor and E. Wyn-Jones, "Electrochemical studies associated with the micellization of cationic surfactants in aqueous mixtures of ethylene glycol and glycerol," Langmuir, vol. 9, no.1, 110-112 1993

[9] G. A. Cookey, C. C. Obunwo and D. O. Uzoma, "The effect of temperature on the micellization of an anionic surfactant in mixed solvent systems," IOSR J. Appl. Chem., vol. 8, no. 12, pp. 49-54, 2015.

[10] S. M. Alawi and M. S. Akhter, "Effect of n-methyl acetamide on the critical micelle concentration of aqueous solutions of some surfactants," J. Korean Chem. Soc., vol. 55, no. 2, pp. 163-168, 2011.

[11] S.E. Olaseni,N.A. Oladoja, I.A. Ololade, C.O. Aboluwoye, M.O. Osundiya "Micellisation of Cetyltrimethyl Ammonium Bromide in Aqueous -organic Media," Chem. Sci. Journal, vol. 52, pp. 1-11, 2012.

[12] A. D. Fenta, "Surface and thermodynamic studies of micellization of surfactants in binary mixtures of 1,2-ethanediol and 1,2,3-propanetriol with water," Int. J. Phys. Sci., vol. 10, no. 8, pp. 276-288, 2015.

[13] K. Prajapati and S. Patel, "Micellization of Surfactants in Mixed Solvent of Different Polarity," Arch. Appl. Sci. Res., vol. 4, no. 1, pp. 662-668, 2012.

[14] A. Rodrı'guez, M. M. Graciani, G. Fernández and M. L. Moyá, "Effects of glycols on the thermodynamic and micellar properties of TTAB in water," J. Colloid Interface Sci., vol. 338 no. 1, pp.207-215, 2009.

[15] T. Nasiru, L. Avila and M. Levine, "Determination of Critical Micelle Concentrations Using UV-Visible Spectroscopy," J. High Sch. Res., vol. 2, no. 1, pp. 1-6, 2011.

[16] Ç. Batigöç, H. Akbaș and M. Boz, "Micellization behaviour and thermodynamic parameters of 12-2-12 gemini surfactant in (water + organic solvent) mixtures," J. Chem. Thermodyn., vol. 43, no. 9, pp. 1349-1354, 2011.

[17] Deepti and K. K. Ghosh, "Micellization behavior of [ $\left.\mathrm{C}_{16}-4-\mathrm{C}_{16}\right], 2 \mathrm{Br}-$ gemini surfactant in binary aqueous-solvent mixtures," Indian J. Chem., vol. 48 A, pp. 1522-1526, 2009.

[18] H. Naorem and S. D. Devi, "Conductometric and surface tension studies on the micellization of some cationic surfactants in water-organic solvent mixed media," J. Surf. Sci. Technol., vol. 22, no. 3-4, pp. 89-100, 2006.

[19] J. Aguiar, J.A. Molina-Bolıvar, J.M. Peula-Garcıa, C.C. Ruiz, "Thermodynamics and micellar properties of tetradecyltrimethylammonium bromide in formamide - water mixtures," journal of Colloid and Interface Sci.,vol.255, pp. 382-390,2002.

[20] A. Kumar and V. Sharma, "Thermodynamics of micellization of ionic surfactants (SDS and CTAB) in aqueous mixtures of PEG-4000," Int. J. ChemTech Res., vol. 8, no. 9, pp.438-443, 2015.

[21] P.K. Sansanwal,"Effect ofCo-solutes on Physicochemical Properties of Surfactant solutions,"J. Sci. industrial Res.,vol.65, pp. 57-64, 2005.

[22] S.K.Shah,S.K.Chatterjee, A. Bhattarai, "Micellization of cationic surfactants in alcohol - water mixed solvent media," Journal of Molecular Liquids,vol. 222, p. 906-914, 2016.

[23] Kabir-ud-Din and P.A. Koya, "Effects of acetonitrile on the micellization and thermodynamic parameters of tetradecyltrimethylammonium bromide: Conductometric and fluorimetric studies," J. Molecular Liquids, vol. 158, no.2,111-116. 2011.

[24] M. M. Islam, M. R. Rahman and M. N. Islam, "Micellization behavior and thermodynamic properties of N-Alkyl Trimethylammonium Bromide surfactants in aqueous media at different temperatures," Int. J. Sci. Eng. Res., vol. 6, no. 1, pp. 1508-1516, 2015. 